\title{
Selected compounds isolated from Morinda citrifolia modulate prostate cancer cell growth
}

\author{
V. Nitteranon ${ }^{1}$, J. Cai ${ }^{2}$, J. Xu ${ }^{2}$, R. G. Godbee ${ }^{3}$, K. L. Parkin ${ }^{1}$ and B. J. Darien ${ }^{1}$ \\ ${ }^{1}$ Department of Food Science, College of Agricultural and Life Sciences, ${ }^{2}$ Department of Medical Sciences, School of \\ Veterinary Medicine, University of Wisconsin, Madison, WI, USA and ${ }^{3}$ Department of Animal Biotechnology/Veterinary \\ Medicine, University Nevada, Reno, NV, USA
}

Morinda citrifolia (noni) fruit is a well-recognised natural product that reportedly has a broad range of immune enhancing effects ${ }^{(1)}$. Although several classes of metabolites have been described, few studies have evaluated the effects of compounds isolated from noni fruit on immune function ${ }^{(2)}$. Of particular interests were two classes of compounds, coumarins and flavanoids. Scopoletin is a pharmacologically active coumarin compound, which has been isolated from noni fruit. Reagent grade scopoletin inhibits the production of inflammatory cytokines. Flavanoids, including rutin, which is in noni, and quercetin, which is the aglycon form of rutin, have recognised antioxidant, anti-inflammatory and antineoplastic properties. The objective of this study was to investigate the dose response of two extraction fractions from noni puree, containing scopoletin and flavanoid compounds, on nitric oxide induction in RAW cells and cell viability in prostatic cancer (PC3) cells. Cell viability was quantified by Calcein AM, MTT and propidium iodide (PI) staining assays. Noni powder was extracted with ethyl acetate (EA) for compound isolation. The crude extract was fractionated using flash column chromatography with $30 \%$ acetone/hexane to obtain four fractions and F3 was identified as scopoletin. Fraction F4 was fractionated further using reversed-phase TLC with $50 \%$ acetone/water to obtain 5 fractions (F4.1, F4.3, F4.4, F4.5 and F4.6). F4.4 was identified as flavonoid-like compound (Fig. 1)

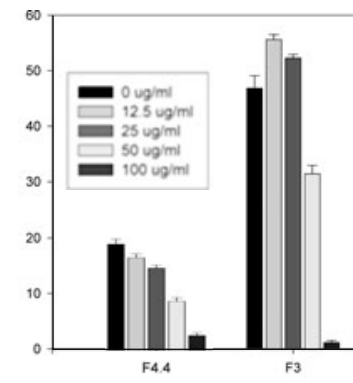

Fig. 1. NO production. Dose response of LPS-induced NO production to F3 and F4.4 fraction in RAW cells. F3 (scopoletin) $\mathrm{IC}_{50}=72.4 \mu \mathrm{g} / \mathrm{ml} ; \mathrm{F} 4.4$ (Flavanoid) $\mathrm{IC}_{50}=79 \mu \mathrm{g} / \mathrm{ml}$. F3 decreased the production of nitrite in RAW cells when activated with LPS.F4.4 exhibited anti-inflammatory activity in LPS-stimulated RAW cells.

Figures 2-4: PC3 cell viability.

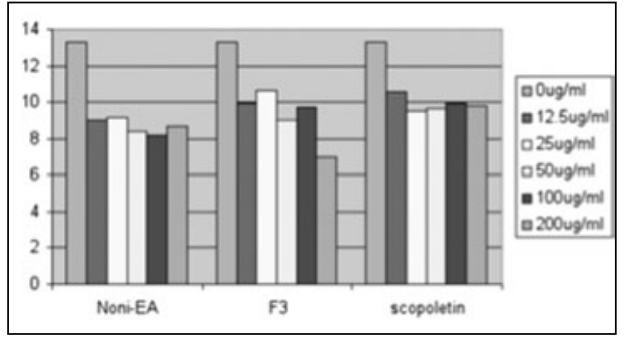

Fig. 3. Effects of different concentrations of noni EA, F3 (scopoletin rich) and reagent scopoletin on PC3 viability (Calcein AM). The EA fraction inhibited PC3 cell growth by about $30 \%$ across all concentrations, while F3 showed greater inhibition $(45 \%)$ at $200 \mu \mathrm{g} / \mathrm{ml}$.

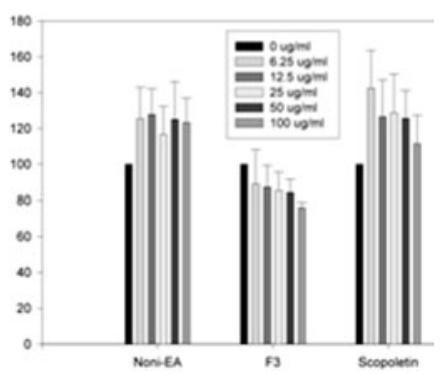

Fig. 2. Effects of different concentrations of noni EA, F3 (scopoletin rich) and reagent scopoletin on PC3 viability (MTT). No cytotoxicity was observed below $100 \mu \mathrm{g} / \mathrm{ml}$.

These preliminary studies demonstrate that scopoletin and flavanoid rich fractions from noni puree inhibited NO production and PC3 cell growth. Further investigations of the mechanism by which different noni compounds singularly or in combination modulate cancer growth is underway.

1. Pawlus AD \& Kinghorn AD (2007) J Pharm Pharmacol 59, 1587-1609.

2. Potterat O \& Hamburger M (2007) Planta Med 73, 191-199. 